\title{
Reconstruction automatique des entités d'un modèle BREP à partir d'un maillage déformé
}

\author{
Borhen Louhichi* — Abdelmajid BenAmara* \\ Vincent François** \\ * Laboratoire de Génie Mécanique Lab-Ma-05 \\ Ecole Nationale d'Ingénieurs de Monastir \\ Av IbnEljazzar 5019 Monastir, Tunisie \\ ** Département de Génie Mécanique, Université du Québec à Trois-Rivières \\ CP 500, Trois-Rivières, Québec, G9A 5H7, Canada \\ lborhen@gmail.com
}

RÉSUMÉ. De nos jours, l'intégration entre les différentes étapes de modélisation d'un produit mécanique est une nécessité afin de réduire le temps de conception et d'optimisation, de valider rapidement de nouveaux concepts et ainsi de gagner en coût, en délai et en qualité. Cela se traduit par une meilleure communication entre les différents outils de CAO et d'analyse. Cette tendance nécessite l'intégration des données entre les différentes tâches et l'unicité du modèle numérique. Cette intégration doit avoir le sens large du terme, c'est-à-dire de la conception vers le calcul et inversement. Notre activité de recherche tente à améliorer cette intégration à travers le développement d'un outil de reconstruction du modèle $C A O$ à partir des résultats de simulation (maillage déformé). Ce papier présente des techniques de reconstruction des entités géométriques et topologiques du modèle $C A O$ à partir d'un maillage déformé dans la démarche de retour des résultats de calcul éléments finis vers la $C A O$.

ABSTRACT. Nowadays, the integration of the different steps of mechanical design process (Design, analysis and manufacturing) is a necessity to reduce the time of design and optimization. This tendency of integrating CAD/CAM/Analysis and automation of the corresponding processes requires shared data between the various tasks using an integrated product model. Our research is oriented to CAD/CAM/Analysis integration by rebuilding the $C A D$ model (BREP) starting from the FE results (deformed mesh). This paper presents techniques for rebuilding the geometrical and topological entities of the CAD model starting from a deformed mesh.

MOTS-CLÉS : intégration, simulation, calcul, reconstruction, CAO, BREP.

KEYWORDS: integration, simulation, analysis, reconstruction, CAD, BREP.

DOI:10.3166/EJCM.18.177-194 (c) 2009 Lavoisier, Paris 


\section{Introduction}

La conception d'un produit mécanique est une activité dynamique qui a pour objectif de répondre à des besoins spécifiés par un cahier des charges. C'est une activité aux multiples facettes qui ne se borne pas uniquement à la définition d'un produit mais qui interfère également avec les processus associés. Ainsi, la définition d'un produit nécessite l'intervention de différents métiers (fabrication, analyse...) qui permettent d'aboutir à une description globale de celui-ci. Les interactions entre métiers qui étaient jusqu'à présent encore très procédurales, c'est-à-dire qui se bornaient à suivre une organisation et une planification des tâches relativement figées, tendent à devenir plus coopératives et dynamiques. Le but est d'assurer une meilleure intégration entre ces différents métiers (Fine, 2001).

En pratique, lors de la conception d'un produit mécanique, le concepteur passe par trois étapes principales:

- la modélisation, avec l'outil de Conception Assistée par Ordinateur (CAO), qui donne le modèle géométrique de la pièce ;

- le calcul (dans la plupart des cas éléments finis (EF)), qui permet de valider ou d'invalider la solution de conception proposée ;

- le développement d'un modèle numérisé de la pièce et l'évaluation et la réparation des données de $\mathrm{CAO}$ de manière à pouvoir les exploiter pour des fonctions de fabrication (Xu et al., 2002 ; Huang et al., 2005).

En conception intégrée ces tâches ne doivent pas êtres vues comme un processus organisé et hiérarchisé dans le temps, mais comme un ensemble de tâches dynamiques en interaction. Pour satisfaire cela, il devient important de proposer un modèle unique qui supporte ces trois spécialités (CAO et Calcul) afin d'améliorer les échanges bidirectionnels entre eux.

Le passage direct (de la $\mathrm{CAO}$ vers le Calcul) se fait le plus souvent à travers des formats standard (IGES, STEP, etc.) ou spécifiques (traducteurs d'un logiciel à un autre). Le passage inverse (retour de Calcul vers la CAO) est quasiment inexistant. Lorsque la simulation E.F est effectuée, s'il y a lieu, l'expert use de son savoir-faire pour exploiter les résultats de calcul (iso valeurs, tableaux de valeurs etc.) et indiquer au concepteur la nature des modifications à effectuer.

Dans le cadre d'un programme de recherche qui consiste à proposer un modèle unique de conception $\mathrm{CAO} / \mathrm{FAO} / \mathrm{Calcul}$, notre tâche consiste à développer le passage inverse (de calcul vers la $\mathrm{CAO}$ ) et concevoir un modèle géométrique déformé comme résultat issu du calcul E.F. L'enjeu des recherches est d'obtenir un modèle dynamique et intégré c'est-à-dire que l'ensemble des informations puissent varier au cours du temps tout en restant compatibles entre elles afin de rendre les simulations numériques aussi automatiques et aussi bien intégrées aux exigences des cycles de conception que possible. 
Pour l'automatisation du passage direct (la modification automatique du modèle de calcul lors d'un changement du modèle CAO) des solutions ont été développées dans ces dernières années (François et al., 2000 ; Shephard, 2000 ; Fine, 2001). Pour le passage inverse, il est envisageable de rendre exploitable les résultats de calcul (déformé) dans l'environnement de CAO en vue d'étudier les assemblages dans leur configuration déformée (configuration de fonctionnement) et la simulation en temps réel des processus de conception et/ou fabrication de produit mécanique.

La reconstruction du modèle géométrique $(\mathrm{CAO})$ repose sur un modèle de type BREP (Boundary Representation) (Mortenson, 1985) qui est une modélisation basée sur les frontières (faces, contours, arêtes et sommets) (figure 1). Le modèle BREP est constitué d'informations topologiques et géométriques. Les informations topologiques existantes au sein d'un modèle BREP sont les connectivités, les liens et les orientations des différentes entités (faces, contours, arêtes et sommets). Les informations géométriques au sein du modèle BREP se résument dans les natures géométriques des surfaces et courbes porteuses des différentes faces et arêtes (face plane, courbe Circulaire...).

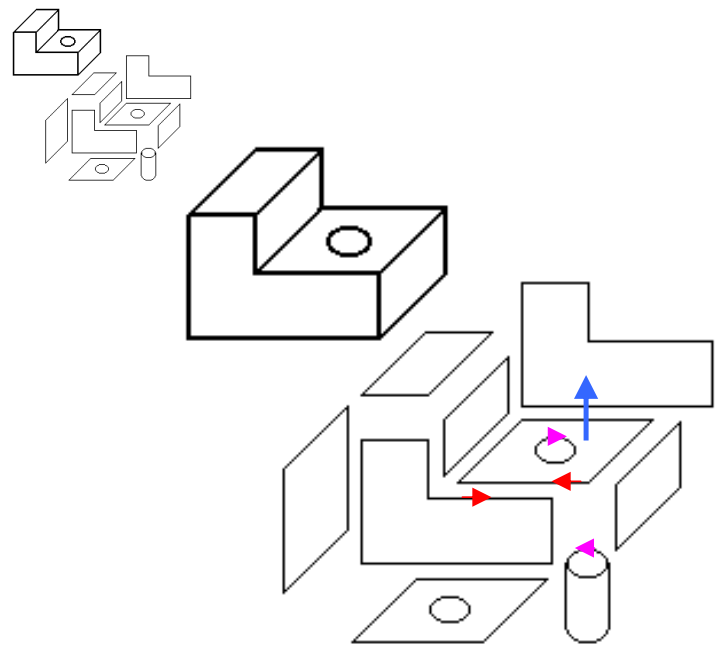

Figure 1. Modèle BREP

Le modèle est choisi pour plusieurs raisons :

- son unicité (pour une pièce mécanique, il existe un seul modèle BREP) ;

- sa description est proche des besoins du mécanicien pour le calcul et la fabrication ;

- sa disponibilité sur la plupart des systèmes CAO. 
Dans cet article, des techniques de reconstruction des entités géométriques et topologiques d'un modèle BREP à partir d'un maillage déformé issu d'un résultat de calcul sont présentées.

\section{Utilité de la reconstruction d'un modèle $\mathrm{CAO}$ à partir des résultats d'une analyse éléments finis}

La prise en compte des résultats de calcul dans l'environnement de conception $\mathrm{CAO}$ pourra pallier un certain nombre de problème de communication entre la $\mathrm{CAO}$ et le Calcul. En effet le libre échange entre CAO et calcul permet de concevoir un modèle intégré (CAO/Calcul) pour la simulation et l'observation.

Un modèle de simulation est un modèle calculatoire du modèle mécanique. Il précise les procédures de calcul, analytiques ou numériques, susceptibles de produire une évaluation des comportements du système étudié. Le modèle d'observation présente un ensemble de variables d'observation du comportement mécanique du produit que le concepteur souhaite analyser pour évaluer le comportement de la solution retenue. Il permet aussi de valider les hypothèses faites au niveau de la construction du modèle mécanique. Ces variables d'observation sont déterminées à partir de la simulation (Aifaoui, 2003).

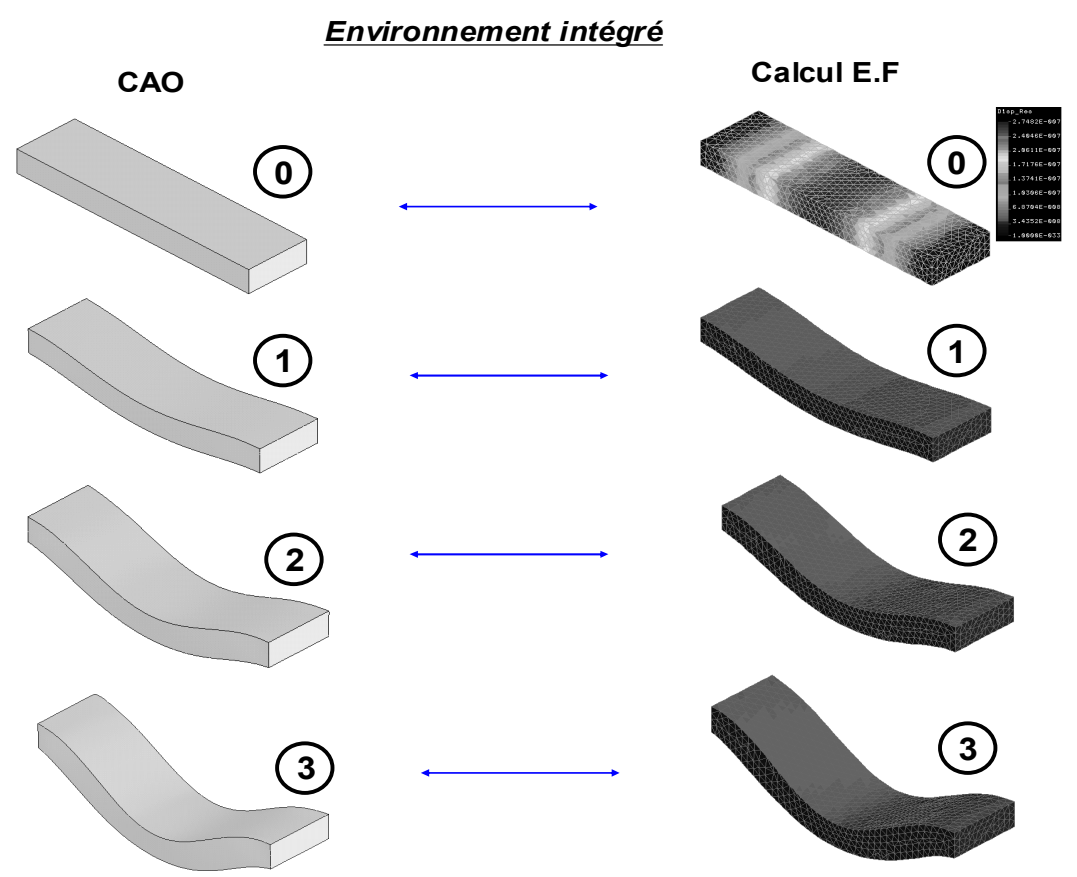

Figure 2. Simulation en configuration réelle d'une étude élastoplastique 
La reconstruction du modèle $\mathrm{CAO}$ à partir des résultats éléments finis (maillage déformé) facilite le libre échange entre conception et calcul et rend possible la visualisation et la simulation du comportement d'un assemblage à l'état déformé (état normal de fonctionnement) afin de détecter des éventuelles interférences (Louhichi et al., 2005). Actuellement les systèmes CAO traitent les assemblages dans leurs configurations rigides. De plus, la reconstruction permet la simulation en configuration réelle (figure 2) des processus de conception et/ou fabrication de produit mécanique (simulation du processus d'emboutissage par exemple). Autour des déformations permanentes de la pièce, le modèle $\mathrm{CAO}$ est reconstruit, le maillage est recalculé et les conditions aux limites sont repositionnées sur la nouvelle forme du modèle.

Les besoins industriels dans ce domaine sont très clairs : disposer d'outils rendant les simulations numériques aussi automatiques et aussi bien intégrées aux contraintes des cycles de conception que possible (Fine, 2001) afin de valider rapidement de nouveaux concepts et ainsi de gagner en coût, en délai et en qualité.

\section{Reconstruction d'un modèle $\mathrm{CAO}$ à partir des résultats d'une analyse éléments finis : algorithme développé}

Dans l'algorithme général de reconstruction (figure 3), les éléments topologiques $\mathrm{du}$ modèle BREP (faces, contours, arêtes et sommets) avec leurs supports géométriques (surfaces, courbes et points) sont reconstruits un par un. Lors de cette reconstruction plusieurs questions peuvent se poser : est-ce que le modèle a changé de topologie (nombre de faces, connectivité entre les entités topologiques...) par rapport à celui avant la déformation ? Est ce que la nature de la face (plane, cylindrique...) a changé ? Plusieurs cas aussi peuvent être rencontrés : la face contient-elle des contours intérieurs ou non ? Faut-il conserver la face non déformée ou en reconstruire une nouvelle?

Connaissant la complexité du problème de reconstruction et la diversité des cas traités en Calcul E.F, l'algorithme général doit être flexible et dynamique afin d'englober les différents cas traités. Dans cet algorithme, la première phase consiste à chercher la topologie et à identifier les différentes entités géométriques du modèle BREP. La deuxième phase, consiste à reconstruire ces entités identifiées précédemment. Dans cette deuxième phase deux cas peuvent être envisagés :

- cas des petites déformations (petites perturbations) : dans ce cas, le nombre d'entités du modèle BREP ainsi que leurs natures géométriques sont connus (identiques au modèle initial). Par exemple le cas d'une poutre soumise à une sollicitation de traction, conserve sa géométrie et sa topologie même si sa dimension latérale change. En fonctionnement réel, cela peut influencer les ajustements et les jeux fonctionnels existant dans un mécanisme. La prise en compte de cette déformation dans le modèle $\mathrm{CAO}$ permet de prévoir les cas de dysfonctionnement. Cependant au niveau de la reconstruction les paramétrisations (point d'insertion, 
rayon, vecteur directeur...) des entités topologiques sont mises à jour par rapport à celles avant déformation (Louhichi et al.,, 2005).

- cas des grandes déformations : c'est le cas le plus complexe, dans lequel les faces changent de géométrie. C'est dans cette phase que l'algorithme doit être dynamique afin d'englober les divers cas rencontrés.

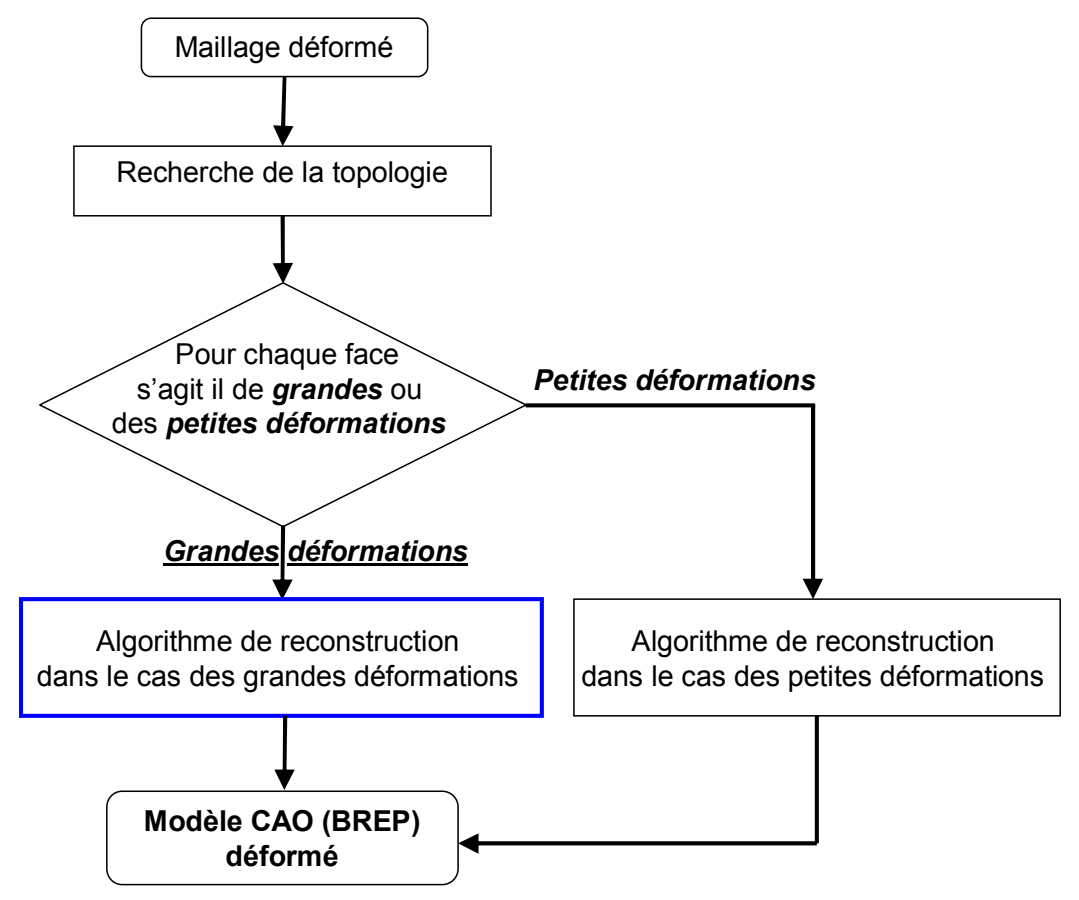

Figure 3. L'algorithme général de reconstruction

\section{Reconstruction des entités d'un modèle BREP : état de l'art}

Le modèle BREP est une description par les frontières d'un solide. Sa reconstruction revient à reconstruire ses frontières (faces, contours, arêtes et sommets). Pour reconstruire les faces et les arêtes, il faut commencer avant tout par construire leurs surfaces et courbes supports. Ces dernières sont évaluées, paramétrées et reconstruites à partir du maillage déformé (nœuds, segments et triangles).

Depuis plusieurs années, plusieurs recherches sont faites sur la reconstruction des courbes et surfaces approximatives à partir d'un nuage de points. (Kruth et al., 1998) et (Yin, 2004) ont développé une méthode de reconstruction d'une surface à partir d'un nuage de points (dans la plupart des cas réguliers) issu d'une machine à commande numérique. Dans le même contexte, d'autres travaux ont développé de nouvelles 
techniques de paramétrisation des courbes et surfaces NURBS (Piegl et al., 1997 ; Jung et al., 2000) à partir d'un nuage de points (figure 4).

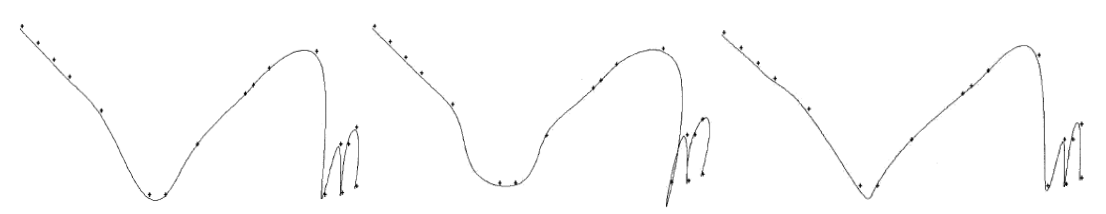

(a)
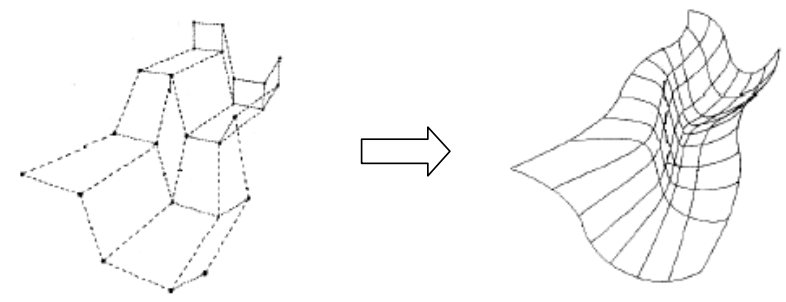

(b)

Figure 4. (a) Différentes techniques de construction d'une courbe à partir d'une séquence de points, (b) construction d'une surface NURBS

D'autres travaux sont basés sur les triangles de Béziers pour l'évaluation d'une surface à partir d'un maillage. Dans ces travaux une surface triangulaire est calculée (triangle de Béziers) à partir de chaque triangle du maillage, de telle façon qu'elle soit continue avec les surfaces voisines (triangles voisins du maillage). La continuité est de type $C^{l}$ ou $C^{2}$ (Walton et al., 1996 ; Kashyap, 1998 ; Morvan et al., 2002 ; Wang, 2004 ; Owen et al., 2002).

En 1998 (Volpin et al., 1998) ont développé un algorithme de reconstruction d'une surface NURBS à partir d'un maillage. La méthode est basée sur trois phases : la simplification du modèle maillé initial, la création d'un maillage quadrilatéral du modèle, la construction des surfaces lisses à partir du maillage quadrilatéral. En 2004, Sarraga (2004), a développé une technique, de modification d'une surface par déplacement des points appartenant à cette surface qui est basée sur une méthode énergétique.

Dans le même contexte d'évaluation, de paramétrisation ou de reconstruction d'une surface à partir d'une triangulation (maillage triangulaire), des travaux se sont intéressés à l'approximation d'une surface par subdivision successive du maillage correspondant (Ren et al., 2003 ; Yang, 2005). Un nouveau nœud est inséré entre deux nœuds consécutifs, afin que chaque arête soit divisée en deux et chaque triangle soit divisé en quatre triangles plus fins tout en respectant la forme de l'objet maillé. D'autres travaux sont basés sur des différents schémas de subdivisions connues 
(schéma de Catmull-Clark, schéma de Loop, schéma de Butterfly...), pour raffiner un maillage successivement en vue d'obtenir une surface lisse (figure 5) (Weiyin et al., 2004 ; Rypl et al., 2004).

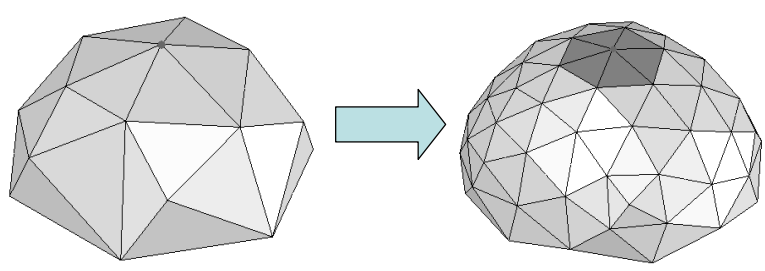

(a)

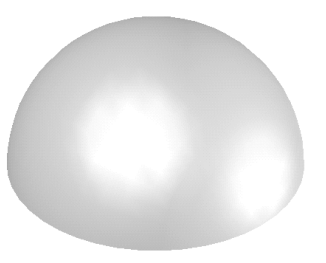

(b)

Figure 5. (a) Subdivision successive, (b) surface lisse

D'autres travaux se sont intéressés à la recherche de la topologie (les orientations, les connectivités...) des différentes entités topologiques d'un modèle BREP (Krysl et al., 2001 ; Park et al., 2003 ; Owen et al., 2001 ; 2003).

Les différents travaux développés au cours du temps, autour de l'évaluation et de la construction des courbes et des surfaces à partir d'un maillage triangulaire, se sont intéressés à l'évaluation de la surface localement au voisinage d'un nœud ou un triangle du maillage. Notre problématique de recherche est l'évaluation et la reconstruction de la surface porteuse d'une face du modèle CAO à partir de la triangulation correspondante. Nous avons adaptés ces méthodes pour obtenir une formulation surfacique globale sous forme de NURBS.

\section{Reconstruction des entités topologiques d'un modèle BREP}

Un modèle BREP est une description géométrique et topologique des frontières d'un objet mécanique. Il est composé par des faces qui possèdent des surfaces porteuses et qui sont limitées par des contours, chacun de ces derniers est composé par des arêtes. Chaque arête possède une courbe porteuse et est limitée par deux sommets. Pour obtenir le modèle BREP, chacune de ces entités (face, arête et sommet) doit être reconstruite tout en suivant la hiérarchie mentionnée (figure 6).

\subsection{Reconstruction des surfaces porteuses des faces}

La complexité de la reconstruction de la surface porteuse d'une face à partir d'une triangulation issue d'un maillage déformé, se présente lorsque la face contient des contours intérieurs : le contrôle de la surface reconstruite est absent en plein milieu du 
contour intérieur. La reconstruction est résolue suivant deux approches. La première approche est basée sur une surface de COONS (Mortenson, 1985), la deuxième est basée sur les outils disponibles dans notre environnement de développement.

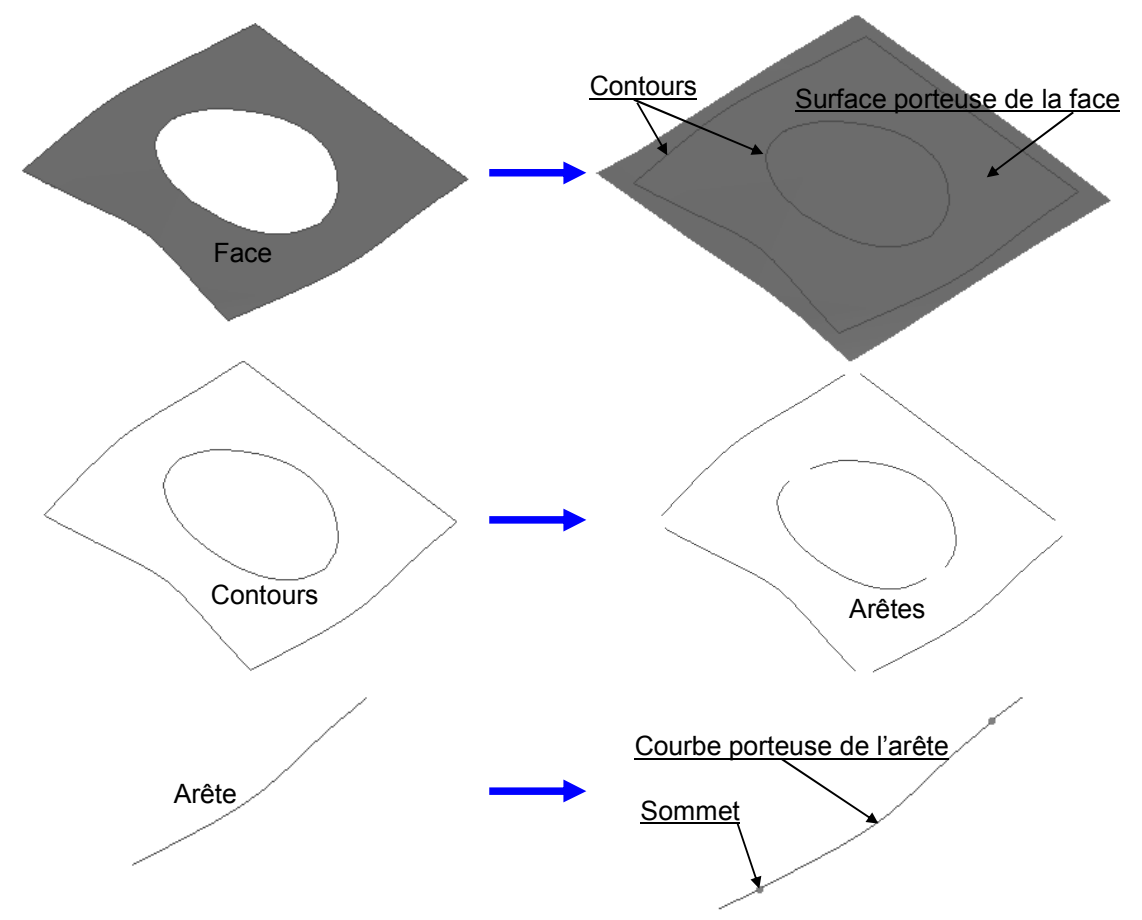

Figure 6. Les entités géométriques et topologiques d'un modèle BREP

\subsection{1. $1^{\text {re }}$ approche}

Cette approche de reconstruction d'une surface porteuse de face à partir d'une triangulation, est basée sur la construction d'un support de points d'une NURBS. Ce support de point est par la suite évalué, suivant deux cas (en utilisant l'algorithme de Walton (Walton et al., 1996) et les surfaces de COONS), pour déterminer la surface déformée. Cette approche de reconstruction permet de transformer le support de points irrégulier (le maillage) en un support de points régulier (les points d'interpolation de la NURBS).

Les étapes de reconstruction suivant cette approche sont les suivantes :

- la construction d'un réseau de points dans l'espace paramétrique de la surface initiale ;

- la détermination de ce réseau dans l'espace réel $(x, y, z)$; 
- projection du réseau de points réels sur le maillage non déformé ;

- le calcul de points d'interpolation de la surface déformée pour obtenir un support de point régulier à partir du support irrégulier qui est le maillage. A cette étape, deux cas se présentent :

$-1^{\text {er }}$ cas : si le point est projeté sur un triangle du maillage, le point d'interpolation de la surface déformée est déterminé par évaluation, en utilisant l'algorithme de Walton (paragraphe suivant), sur le même triangle dans le maillage déformé ;

- $2^{\mathrm{e}}$ cas : si le point est projeté dans le vide (c'est-à-dire le point est au milieu d'un trou sur la surface), le point d'interpolation est obtenu par projection sur la surface de COONS représentée à partir du contour intérieur, de la surface, en question (figure 7c).

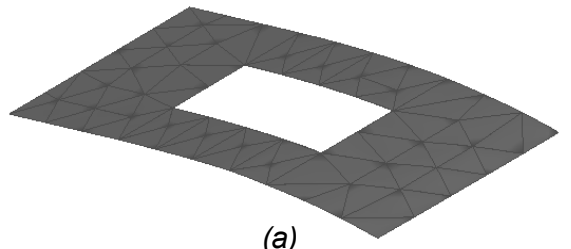

(a)

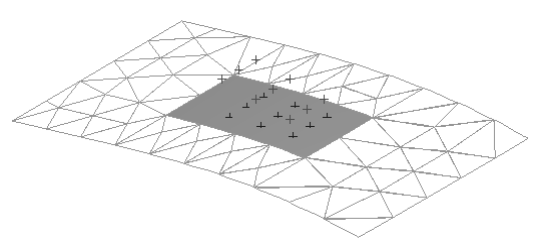

(c)

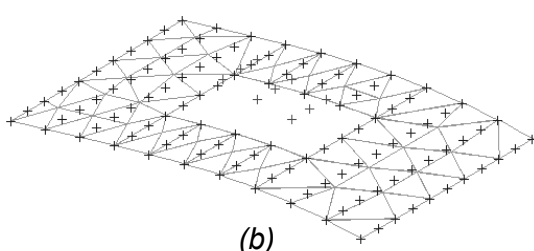

(b)

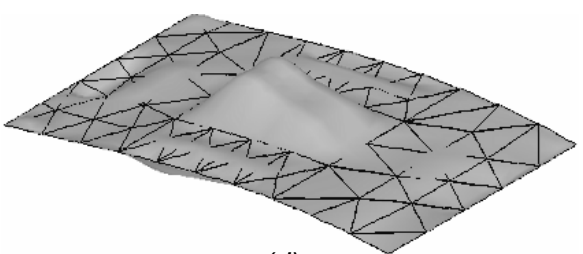

(d)

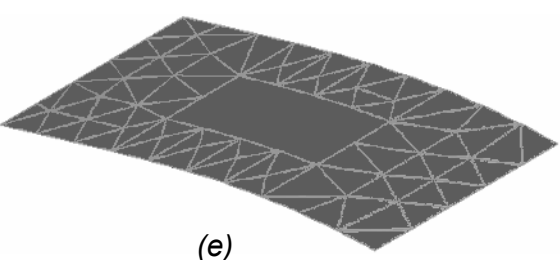

Figure 7. (a) Maillage correspondant à une face déformée, (b) points d'interpolation obtenus par évaluation des triangles du maillage, (c) points d'interpolation obtenus par projection sur la surface COONS, (d) face déformée obtenue sans utilisation de la surface COONS, (e) face déformée obtenue en utilisant la surface de COONS

L'algorithme de (Walton et al., 1996), consiste à évaluer la surface localement pour chaque triangle du maillage (figure 8 ) : 
- calculer les coordonnées locales du point (support non déformé) projeté dans le triangle (maillage non déformé) $\boldsymbol{u}, \boldsymbol{v}$ et $\boldsymbol{w}$;

- insérer un point qui a comme coordonnées $\boldsymbol{u}, \boldsymbol{v}$ et $\boldsymbol{w}$ dans le même triangle de maillage déformé ;

- à partir de ce point inséré, déterminer le point de support d'interpolation déformé sur la surface reconstruite par l'algorithme de Walton (Walton et al., 1996).
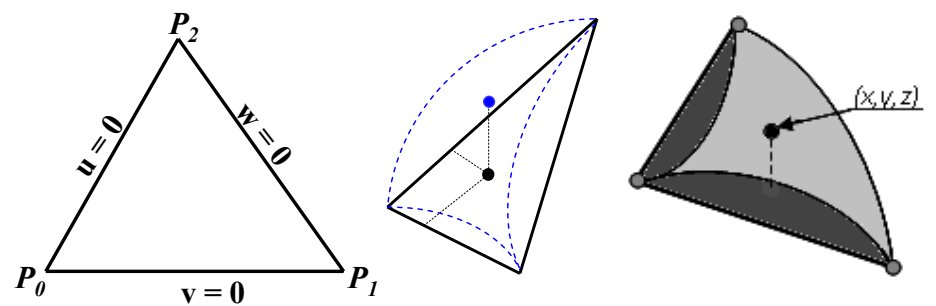

Figure 8. Evaluation d'un point à partir d'un triangle

\subsection{2. $2^{e}$ approche}

Cette deuxième approche est basée sur des outils et des fonctions disponibles dans notre environnement. L'approche est développée sous l'environnement Open Cascade de Dassault Système qui contient des fonctions dans ses API (application programming interface) permettant d'adhérer une surface à un ensemble de courbes et points (figure 9). Ces fonctions de Open Cascade sont basées sur des méthodes énergétiques (Volpin et al., 1998; Sarraga, 2004 ; OpenCascade). Cela correspond bien à notre cas : la surface, qui passe par les nœuds du maillage et qui adhère aux courbes de contours, est reconstruite.

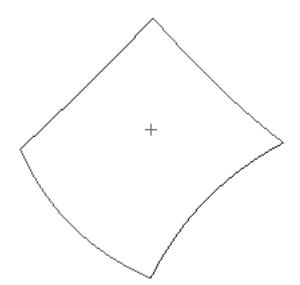

(a)

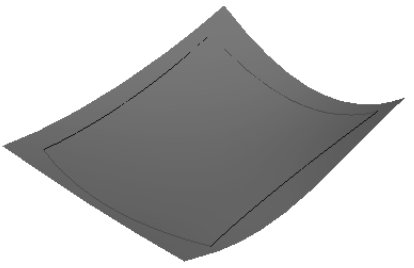

(b)

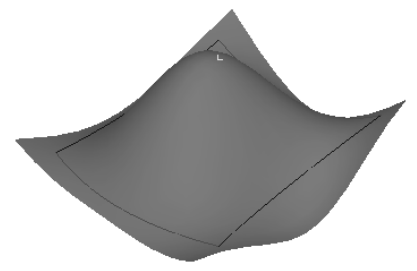

(c)

Figure 9. (a) Les courbes et le point sont contraints, (b) la surface adhère sur les courbes, (c) la surface adhère sur les courbes et passe par un point 


\subsubsection{Méthode énergétique}

La méthode énergétique est une méthode développée au sein de la plateforme Open Cascade (Matra Datavision). Cette méthode possède des données (figure 10) :

- des courbes contraintes (ce sont des courbes reconstruites par interpolation des nœuds de maillage des arêtes dans le modèle déformé) (figure 9);

- des points contraints (issus du maillage de la face) (figure 9).

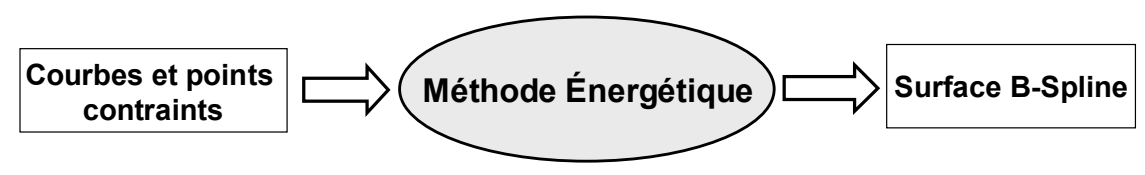

Figure 10. Entrées/sortie de la méthode énergétique

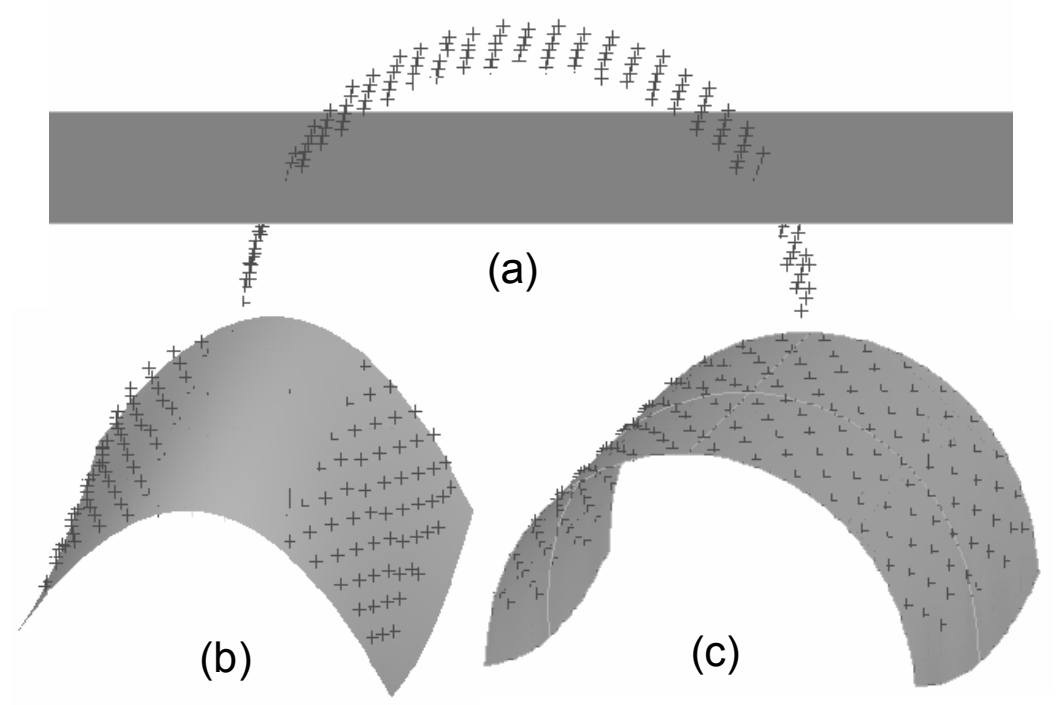

Figure 11. (a) Plan d'inertie, (b) surface initiale, (c) surface finale

Le résultat de cette méthode est une surface (figure 9) qui lisse les différentes contraintes définies précédemment.

Etant donné un ensemble de points et courbes contraints, la construction de la surface NURBS par la méthode énergétique se fait en trois grandes étapes :

$-1^{\text {re }}$ étape : chaque courbe contrainte est discrétisée en un ensemble de points, par la suite le plan d'inertie de l'ensemble des points est construit puis les différentes contraintes sont projetés sur ce plan (figure 11). Le plan d'inertie est le plan qui 
passe par le flux maximum de points, c'est également le plan déterminé par la méthode des moindres carrés ;

$-2^{\mathrm{e}}$ étape : une surface initiale qui est proche de la surface finale est calculée par déformation du plan d'inertie (figure 11). Cette dernière opération se fait en se basant sur un ensemble bien déterminé de points contraints, par minimisation des distances entre ces derniers et leurs projetés sur le plan d'inertie ;

$-3^{\mathrm{e}}$ étape : la surface finale est cherchée (figure 11) à partir de celle trouvée dans la deuxième étape. La construction de la surface se fait sur plusieurs itérations mais dans la plupart des cas une seule itération est suffisante sauf pour des surfaces plus complexes qui présentent des pics et courbures (cas peu fréquent en mécanique du solide).

\subsubsection{Algorithme développé}

L'algorithme de reconstruction d'une surface déformée à partir d'un maillage est le suivant (figure 12).
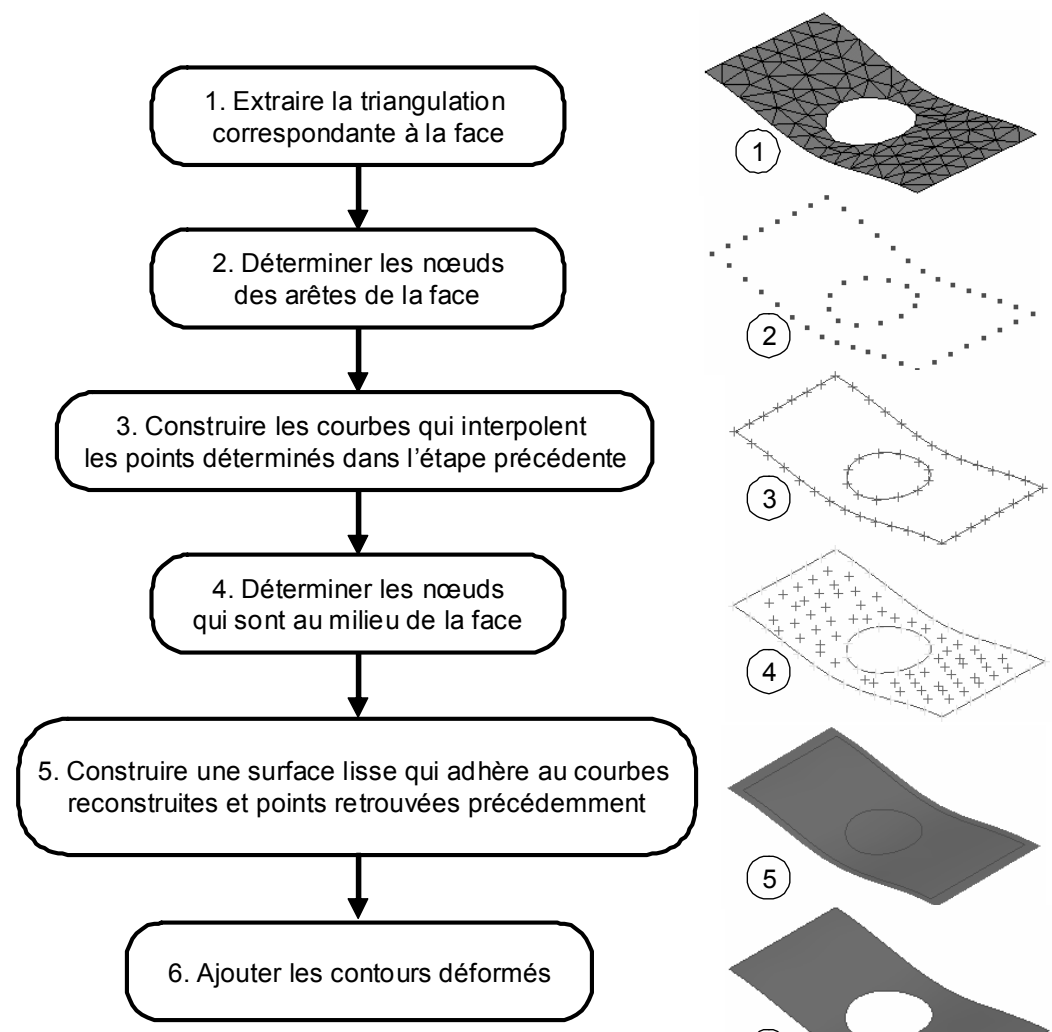

(5)

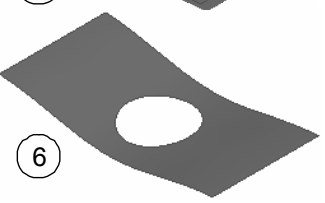

Figure 12. Algorithme de reconstruction d'une surface à partir d'un maillage par la méthode énergétique 


\subsubsection{Synthèse}

Pour la reconstruction des faces d'un modèle BREP deux approches sont présentées (sections 5.1.1. et 5.1.2.) :

- la première approche donne de bons résultats dans le cas où les déformations ne sont pas très importantes. Dès que les déformations deviennent importantes, la méthode peut perdre sa précision puisque la surface reconstruite est le résultat d'une interpolation d'une part, des points qui sont calculés à partir du maillage, d'autre part, des points calculés par projection sur la surface de COONS. Un autre problème d'erreurs numériques entre les faces, apparaît avec cette méthode lors de la reconstruction du BREP au complet, puisque les faces reconstruites ne collent pas parfaitement aux contours.

- la deuxième approche est plus efficace que la première, elle s'applique quelles que soient la nature et l'importance des déformations. Des problèmes apparaissent parfois, lors de la reconstruction, si le maillage est grossier et les informations (points contraints) ne sont pas suffisantes. Ces problèmes se manifestent par l'apparition de régions bombées et par l'apparition de pics sur la surface. Dans ce cas, pour ajouter de l'information dans quelques régions de la surface, il faut mieux raffiner le maillage où insérer des nouveaux points calculés à partir du maillage. Ceci correspond à l'utilisation des surfaces de subdivision. Cette deuxième approche est retenue pour la reconstruction du modèle BREP.

\subsection{Reconstruction des arêtes et contours}

La reconstruction des arêtes est simple. Les noeuds qui correspondent à une arête dans le maillage déformé sont déterminés puis sont interpolés pour obtenir l'arête correspondante. Dans le cas de petites perturbations, l'arête garde sa nature de celle avant déformation (par exemple une arête circulaire reste circulaire) seuls les paramètres changent : vecteur directeur, point d'insertion... (Louhichi et al., 2005).

Dans le cas des déformations importantes, les points d'interpolation des arêtes, issus du maillage, sont déterminées et projetés un par un sur la surface en question. Par la suite les arêtes et contours, qui doivent être dans l'espace paramétrique de la surface, sont reconstruites une par une. A la fin la surface doit être coupée (limitée) suivant ces contours pour obtenir la face (figure 12).

\section{Résultats de reconstruction}

Dans cette illustration, différentes pièces (prismatique, cylindrique) sont soumises à différentes sollicitations (traction, flexion et gonflement). Ces pièces contiennent différents types de faces ainsi que différents types d'arêtes. Les faces et les arêtes sont détectées et reconstruites une par une (sections 5.1.2. et 5.2.). Toutes les surfaces 
porteuses des faces reconstruites et les courbes porteuses des arêtes sont de types NURBS. L'erreur de reconstruction est calculée par projection des points d'interpolation (nœuds du maillage déformé) sur la surface reconstruite.

Ces différentes pièces sont reconstruites dans leurs domaines plastiques entraînant un changement de volume variant entre $3 \%$ et $15 \%$ et un changement de superficie variant entre $5 \%$ et $10 \%$. L'algorithme produit des surfaces assez précises puisque les erreurs sont de l'ordre de $10^{-4}$ faibles par rapport à la dimension de la pièce qui est de l'ordre de $10^{2}$.

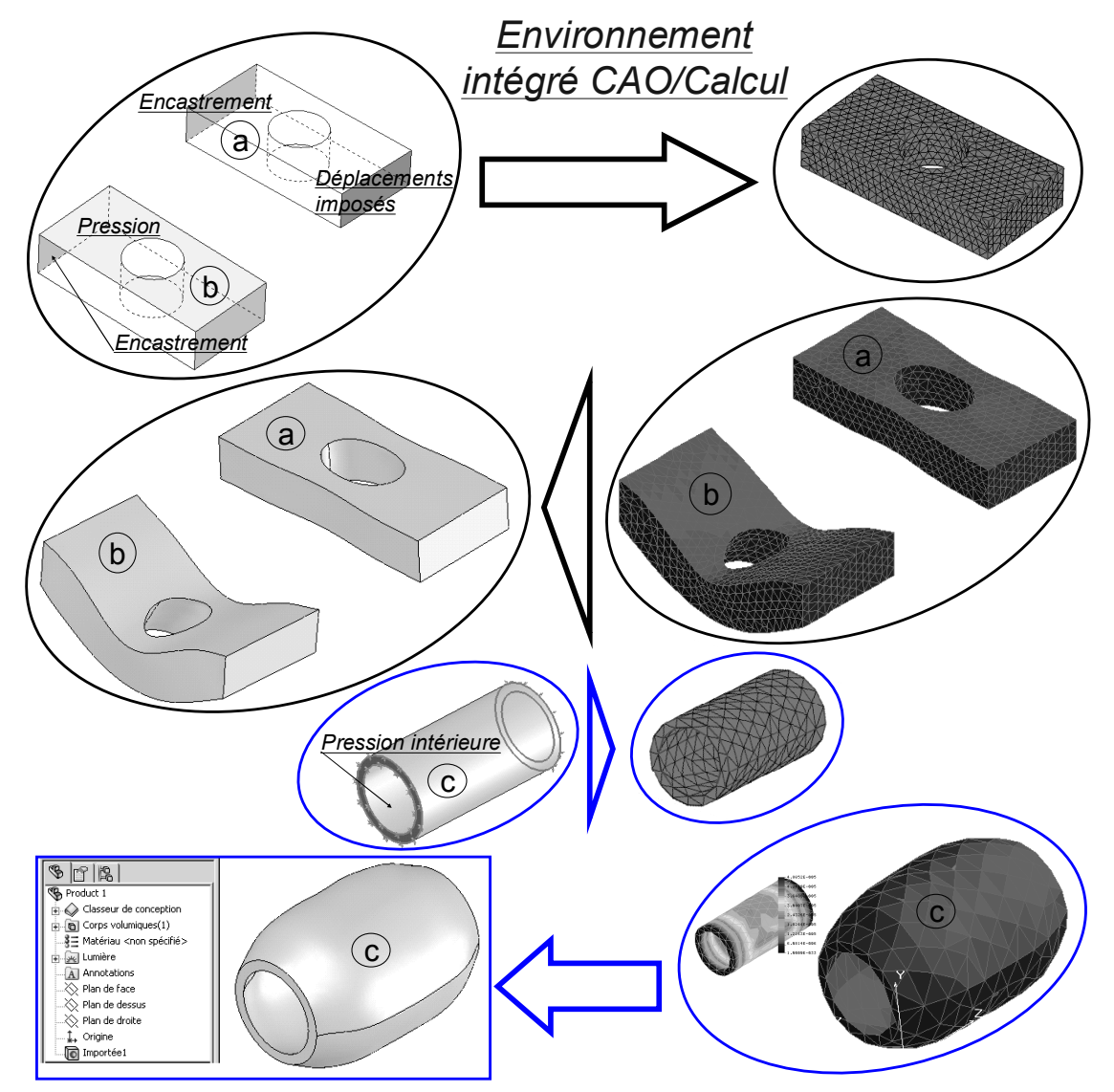

Figure 13. Illustration de la démarche : résultats de reconstruction (a) pièce en traction, (b) pièce en flexion, (c) cylindre sous pression intérieure 


\section{Conclusions et perspectives}

Dans cet article, différentes techniques de reconstruction des entités géométriques d'un modèle BREP, et leurs limites ont été présentées. Les approches présentées traitent la partie géométrique du modèle BREP et supposent que la topologie du modèle n'a pas changé après déformation (le nombre des faces, arêtes et sommets et leurs connectivités restent le même). Les différentes approches et techniques de reconstruction doivent être améliorées en vue d'obtenir un environnement intégré $\mathrm{CAO} / \mathrm{Calcul}$ efficace et dynamique.

Actuellement lors de la reconstruction du modèle BREP, on décide dès le début si la reconstruction se réalise dans l'hypothèse de petites déformations et par la suite les natures des entités géométriques ne changent pas (face cylindrique reste cylindrique après déformation) où bien dans les conditions des grandes déformations et dans ce cas les courbes et surfaces NURBS vont être utilisées.

Les travaux se poursuivent à présent en solutionnant ce dernier problème pour que la reconstruction des faces devienne plus réaliste et complètement automatique. Le modèle BREP doit être dynamique et ses entités doivent être reconstruites suivant l'importance de leurs déformations sans apparition des erreurs numériques entre eux.

\section{Bibliographie}

Aifaoui N., Intégration $\mathrm{CAO} /$ Calcul, une approche par les features de calcul, Thèse de Doctorat, Université de Valenciennes, Juillet 2003.

Fine L., Processus et méthodes d'adaptation et d'idéalisation de modèles dédiés à l'analyse de structures mécaniques, Thèse de doctorat, Institut nationale polytechnique de Grenoble, 2001.

Francois V., Cuillère J.C., "3D Automatic remeshing applied to model modification", Computer-Aided Design, vol. 32, n ${ }^{\circ}$ 7, 2000, p. 433-444.

Joon C. Park, Yun C.Chung, "A tolerant approach to reconstruct topology from unorganized trimmed surfaces”, Computer Aided Design, vol. 35, 2003, p. 807-812.

Jung H. B. and Kim K., "A New Parameterisation Method for NURBS Surface Interpolation", The International journal of Advanced Manufacturing Technology, vol. 16, 2000, p. 784-790.

Kruth J.-P., Kerstens A., "Reverse engineering modelling of free-form surfaces from point clouds subject to boundary conditions", Journal of Materials Processing Technology, vol. 76, 1998, p. 120-127.

Louhichi B., BenAmara A., François V., «Intégration CAO/Calcul par reconstruction des modèles CAO à partir des résultats de calcul», Revue Internationale d'Ingénierie Numérique, vol. 1, $\mathrm{n}^{\circ} 1,2005$, p. 9-26. 
Ma Weiyin, Ma Xiaohu, Shiu-Kit Tso, Zhigeng Pan, "A direct approach for subdivision surface fitting from a dense triangle mesh", Computer Aided Design, vol. 36, 2004, p. 525-536.

Mortenson E. Michael, Geometric Modeling, Wiley, New York, 1985.

Morvan J.M., Thibert B., "On the approximation of a smooth surface with a triangular mesh", Computational Geometry, vol. 23, 2002, p. 337-352.

Opencascade www.Opencascade.com.

Petr Krysl, Micheal Ortiz, "Extraction of boundary representation from surface triangulations", International journal for numerical methods in engineering, vol. 50, 2001, p. 1737-1758.

Piegl L.A. and Tiller W., The NURBS book, Springer-Verlag, 1997.

Praveen Kashyap, "Geometric interpretation of continuity over triangular domains", Computer Aided Geometric Design, vol. 15, 1998, p. 773-786.

Ramon F. Sarraga, "Modifying CAD/CAM surfaces according to displacements prescribed at a finite set of points", Computer Aided Design, vol. 36, 2004, p. 343-349.

Ren B.Y., Hagiwara I., "Composite freeform surface reconstruction using recursive interpolating subdivision scheme”, Computers in Industry, vol. 50, n 3, 2003, p. 265-275.

Rypl D., Zdenek Bittnar "Triangulation of 3D surfaces reconstructed by interpolating subdivision”, Computers and Structures, vol. 82, 2004, p. 2093-2103.

Shaoming Wang, "A smooth surface interpolation to 3D triangulations", Journal of computation and applied Mathematics, vol. 163, 2004, p. 287-293.

Steven J. Owen and David R. White, "Mesh based geometry: A systematic approach to constructing geometry from a finite element mesh", $10^{\text {th }}$ International Meshing Roundtable Newport Beach, California, U.S.A. 7-10 October 2001.

Steven J. Owen, David R. White, Timothy J. Tautges, "Facet-based surfaces for 3D mesh generation", $11^{\text {th }}$ international meshing roundtable, 15-18 September 2002, Ithaca, New York.

Steven J. Owen, David R. White "Mesh-based geometry", International Journal for Numerical Methods in Engineering, vol. 58, n² 2, 2003, p. 375-395.

Shephard M.S., "Meshing environment for geometry-based analysis", International Journal for Numerical Methods in Engineering, vol. 47, n 1-3, 2000, p. 169-190.

Volpin O., Sheffer A., Bercovier M. and Joskowicz, "Mesh simplification with smooth surface reconstruction", Computer aided Design, vol. 30, n 11, 1998, p. 875-882.

Walton D. J. and Meek D. S., “A triangular G1 patch from boundary curves”, Computer Aided Design, vol. 28, 1996, p. 113-123.

$\mathrm{Xu}$ X.Y., Wang Y.Y., "Multi-model technology and its application in the integration of CAD/CAM/CAE", Journal of Materials Processing Technology, vol. 129, 2002, p. 563-567.

Xunnian Yang, "Surface interpolation of meshes by geometric subdivision", Computer Aided Design, vol. 37, 2005, p. 497-508. 
194 European Journal of Computational Mechanics. Volume 18 - No. 2/2009

You-Min Huang, Hsiang-Yao Lan, "CAD/CAE/CAM integration for increasing the accuracy of mask rapid prototyping system”, Computers in Industry, vol. 56, 2005, p. 442-456.

Zhongwei Yin, "Reverse engineering of a NURBS surface from digitized points subject to boundary conditions", Computer \& Graphics, vol. 28, 2004, p. 207-212.

Received: 11 September 2008 Accepted: 23 November 2008 